\title{
Propulsion of swimming microrobots inspired by metachronal waves in ciliates: from biology to material specifications
}

\author{
Stefano Palagi, Edwin Jager, Barbara Mazzolai and Lucia Beccai
}

\section{Linköping University Post Print}

\section{Tweet}

N.B.: When citing this work, cite the original article.

Original Publication:

Stefano Palagi, Edwin Jager, Barbara Mazzolai and Lucia Beccai, Propulsion of swimming microrobots inspired by metachronal waves in ciliates: from biology to material specifications, 2013, Bioinspiration \& Biomimetics, (8), 4, 046004.

http://dx.doi.org/10.1088/1748-3182/8/4/046004

Copyright: Institute of Physics: Hybrid Open Access http://www.iop.org/

Postprint available at: Linköping University Electronic Press http://urn.kb.se/resolve?urn=urn:nbn:se:liu:diva-102772 


\title{
Propulsion of swimming microrobots inspired by metachronal waves in ciliates: from biology to material specifications
}

\author{
Stefano Palagi ${ }^{1}$, Edwin WH Jager ${ }^{2}$, Barbara Mazzolai $^{1}$ and \\ Lucia Beccai ${ }^{1}$ \\ ${ }^{1}$ Center for Micro-BioRobotics@SSSA, Istituto Italiano di Tecnologia, 56025 \\ Pontedera, Italy \\ ${ }^{2}$ Biosensors and Bioelectronics Centre, Department of Physics, Chemistry and \\ Biology, Linköping University, 58183 Linköping, Sweden
}

E-mail: stefano.palagi@it.it, lucia.beccai@iit.it

\begin{abstract}
The quest for swimming microrobots originates from possible applications in medicine, especially involving navigation in bodily fluids. Swimming microorganisms have become a source of inspiration because their propulsion mechanisms are effective in the low-Reynolds number regime. In this study, we address a propulsion mechanism inspired by metachronal waves, i.e. the spontaneous coordination of cilia leading to the fast swimming of ciliates. We analyze the biological mechanism (referring to its particular embodiment in Paramecium caudatum), and we investigate the contribution of its main features to the swimming performance, through a three-dimensional finiteelements (FE) model, in order to develop a simplified, yet effective artificial design. We propose a bioinspired propulsion mechanism for a swimming microrobot based on a continuous cylindrical electroactive surface exhibiting perpendicular wave deformations travelling longitudinally along its main axis. The simplified propulsion mechanism is conceived specifically for microrobots that embed a micro-actuation system capable of executing the bioinspired propulsion (self-propelled microrobots). Among the available electroactive polymers (EAPs), we select Polypyrrole (PPy) as the possible actuation material and we assess it for this particular embodiment. The results are used to appoint target performance specifications for the development of improved or new electroactive materials to attain metachronal-waves-like propulsion.
\end{abstract}




\section{Introduction}

There is a growing interest in artificial micro-swimmers, amongst others motivated by the desire to understand the fundamental physics of swimming at low Reynolds numbers, the relevance of swimming in microscale biological processes, as well as the practical desire to build microfluidic pumps and swimming microrobots [1].

Swimming microrobots are mobile, untethered devices that have a typical size in the sub-millimetre range, i.e. from one millimetre down to one micrometre [2], and are able to move within a liquid medium [3]. The envisioned applications of swimming microrobots are mainly found in medicine, especially involving navigation in bodily fluids, such as blood and cerebrospinal fluid, for the execution of very localized diagnosis or therapy tasks [4].

Swimming microrobots typically operate in the low-Reynolds-number regime (low$R e)$, where inertia plays a minor role with respect to viscous damping. Swimming at these scales thus requires mechanisms that are substantially different from those employed at the macroscale [5]. Therefore, designing a swimming microrobot is certainly a challenging task. A viable and effective way to cope with this issue is to exploit Nature as a source of inspiration. Microorganisms are indeed able to effectively swim at the microscale by adopting different techniques [2].

Bacteria are absolutely the most studied biological models in swimming microrobotics. Tiny microrobots (few tens of microns in length) have been developed that swim by means of helical tails resembling bacterial flagella [6,7]. The rotating action by the molecular motor in the microorganism was replaced by an external magnetic torque, wirelessly applied by means of a rotating magnetic field.

Also, in looking for solutions within the eukaryotic realm, an elastic tail vaguely resembling eukaryotic flagella was developed for magnetically propelling a swimming microdevice [8, 9]. Moreover, artificial single cilia mimicking their natural counterpart but relying on magnetic actuation, were investigated both for propulsion of microrobots [10] and for fluid transport in microfluidics [11, 12.

These biological mechanisms of propulsion were also proposed to be implemented in microrobots that could self-propel [13]. The term self-propulsion refers to the capability of an artificial swimming microrobot of propelling its own body through the fluid by means of actions generated within the microrobot itself, rather than relying on external fields for actuation, which is instead the most common situation in state-of-the-art microrobotics. However, heading for self-propulsion significantly increases not only the flexibility and the potentialities, but also the level of complexity of the design and fabrication of microrobots. It is unlikely that the tiny and complex biological propelling appendages could be individually replicated, controlled and actuated in a microscale artificial device. This would be too complex and technologically challenging at this scale. In addition, these tiny external appendages represent a hindrance. They could detach from the microrobot body, get stuck or damage the external environment which is particularly dangerous in medical applications. For this reason, micro-swimmers relying 
on internal mechanisms of propulsion have been recently proposed 14, 15.

Here, we investigate propulsion by metachronal waves in ciliates, in order to take inspiration for designing an artificial mechanism that could be potentially embedded in self-propelled swimming microrobots. Metachronal waves consist of a collective behaviour arising from self-coordination of cilia. This substantially originates propulsive waves of deformation propagating on the surface of the body of the microorganism [1]. The phenomenon of metachronal waves is widespread in nature, being found in ciliates and in all eukaryotic cells that possess arrays of cilia. Taking inspiration from this highlevel behaviour, instead than mimicking the complexity of the actual ciliary propulsion, we propose a novel propulsion mechanism that does not involve external appendages, but that is based on small deformations of the surface of the microrobot. We already investigated metachronal-waves-based propulsion in a preliminary work [16]; here a deeper analysis is reported, with a focus on the conceptual design of a propulsion system for artificial micro-swimmers.

The reference biological mechanism is introduced in Section 2, while in Section 3 a finite-elements (FE) model is developed and adopted for predicting the swimming speed of a microorganism example (Paramecium caudatum). After this preliminary validation, the model is used to investigate the role of the key features of the propulsion mechanism on the final speed of the micro-swimmer (Section 4). This analysis allowed us to define a simplified concept of the propulsion mechanism to be embedded in an artificial swimming microrobot. The design of the artificial embodiment of the metachronalwaves-inspired propulsion mechanism is, therefore, presented in Section 5, in which particular focus is given to the selection of a suitable actuation technology and to the expected performances of the artificial micro-swimmer. Finally, conclusions are reported in Section 6.

\section{Biological mechanism}

Cilia are short appendages (about $10-12 \mu \mathrm{m}$ long) that can be found in densely packed arrays on the external surface of different kinds of eukaryotic cells. These include a group of unicellular microorganisms, which are called ciliates. From a microrobotic point of view, ciliates are interesting because they are the largest and fastest micro-swimmers in nature. Ciliates such as those belonging to the genus Paramecium have typical size of few hundred micrometres and can propel themselves at speeds above one millimetre per second. Indeed, although they do not have long appendages for propulsion, the thousands of short and thin cilia covering their surface exhibit a self-coordinated action that is responsible for the fast propulsion of the whole body of the microorganism through the fluid [1].

The fast swimming of ciliates can only be understood by considering that two levels of symmetry breaking are present in their propulsion. Symmetry breaking is crucial in low-Re swimming, since non-reciprocal motion of the micro-swimmer is needed to achieve a net displacement. In the case of ciliates, first each cilium on the surface of 
a)

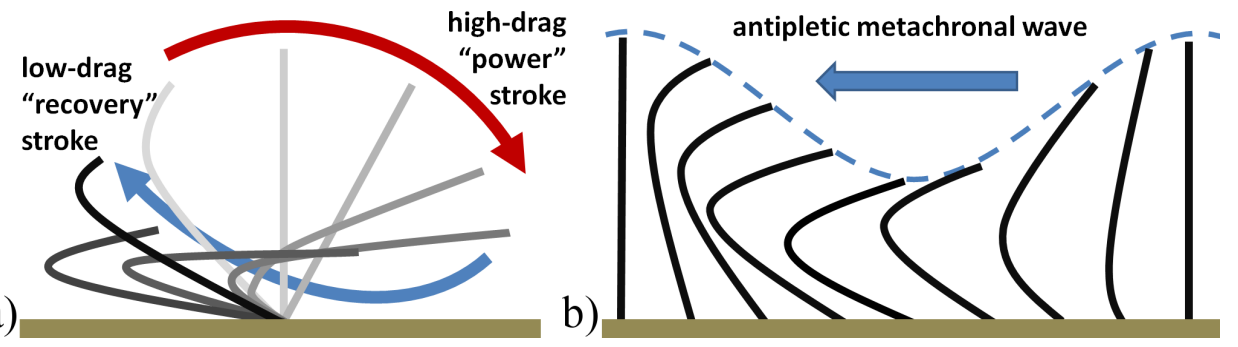

Figure 1. Propulsion of fluids by cilia: a) asymmetric beating pattern of a single cilium, consisting of a high-drag power-stroke and a low-drag recovery stroke (the grayscale corresponds to different position of the cilium in time); b) antipletic metachronal waves in an array of cilia.

the microorganism exhibits a complex asymmetric beating pattern. This non-reciprocal deformation involves a high-drag "power" stroke and a low-drag "recovery" stroke [1, 17] (see Figure 1(a)). Moreover, since cilia are closely packed on the surface, hydrodynamic interactions among them lead to a coordinated collective behaviour, termed metachronal waves [18 (see Figure 1(b)), which is responsible for the second level of symmetry breaking, and, in addition, results in an energetic advantage in ciliary beating [19].

Metachronal waves thus originate from the fact that, just because of hydrodynamic interactions, each cilium beats with a small constant phase difference with respect to its neighbours [1, 20]. Therefore they appear as propagating waves of deformation of the surface of the microorganism. Metachronal waves are very widespread in nature and their descriptive parameters, such as amplitude, wavelength and frequency, present a great variability among the cells that exhibit them. In addition, metachronal coordination may take several forms, depending on the specific organisms and conditions, such as sympletic metachrony (i.e. effective stroke moving in the same direction as the wave crests) and antipletic metachrony (i.e. effective stroke moving in the opposite direction with respect to the wave crests) [17]. Metachronal waves are indeed a very flexible mechanism that allow cells to easily adapt to their particular environment. Nevertheless, their basic working principle and their role in fluid propulsion are independent on the specific biological embodiment. Therefore, propulsion of fluids and propulsion through fluids by means of metachronal waves are a well-known phenomenon that attracted the interest of the biological [21], as well as the mathematical and physical 22] scientific communities. In particular, in the early '50s, Lighthill introduced the so-called envelope model [23], which was further developed by Blake in the '70s 22]. This model predicts the propulsion of ciliates by replacing the individuality of cilia with a representation of metachronal waves as a progressing waving continuous envelope. The envelope model thus highlights the fundamental role of metachronal waves, emphasizing that the self-coordination of cilia is the key factor in the propulsion of ciliates. 


\section{Modelling}

The envelope model is a complex analytical model developed for simplified geometries of the microorganism body. Here, we exploit the basic principle of the envelope model of the propulsion of ciliates, i.e. the progressing waving envelope, for developing a FE model of the metachronal-waves-based propulsion. In addition, the finite-elements implementation allows us to use the model as a flexible tool for designing swimming microrobots inspired by ciliates, as reported in Section 4 .

\subsection{Model description}

The liquid in which the micro-swimmer moves was considered as an incompressible fluid in the laminar regime. Thus it is governed by the Navier-Stokes equations [1], namely

$$
\rho_{f}\left[\frac{\partial \boldsymbol{u}}{\partial t}+(\boldsymbol{u} \cdot \nabla) \boldsymbol{u}\right]=-\nabla p+\eta_{f} \nabla^{2} \boldsymbol{u}+\boldsymbol{f}_{e}, \quad \nabla \cdot \boldsymbol{u}=0
$$

where $\rho_{f}$ and $\eta_{f}$ are, respectively, the mass density and the dynamic viscosity of the fluid, $\boldsymbol{f}_{e}$ accounts for external forces and $\boldsymbol{u}$ and $p$ represent the velocity vectorial field and the pressure scalar field, respectively. It is noteworthy that the full formulation was adopted, rather than the simplified version for $R e \ll 1$ (Stokes equations), since in the actual modelled conditions $R e \approx 1$.

The micro-swimmer was considered as a prolate spheroid moving along its major axis in a liquid environment without boundaries, such as walls (in contrast to e.g. the motion within a channel). As a matter of fact, the prolate spheroid is a good approximation of the shape of the body of many ciliates, such as those of the genus Paramecium [24] (see Figure 2). The spheroid can be defined by the expression of its surface that, in a cylindrical reference frame $r \phi z$ (adopted only as an auxiliary reference system for defining the variables related to the micro-swimmer), reads

$$
\frac{r^{2}}{b^{2}}+\frac{z^{2}}{a^{2}}=1
$$

where $a$ and $b$ are the major and minor semi-axes, respectively.

In order to model the deformation of the surface we also adopted an auxiliary coordinate defined as

$$
\zeta(r, z)=\frac{p_{F_{-}}(r, z)-p_{F_{+}}(r, z)}{d_{F}} \frac{\gamma}{4}
$$

where $p_{F_{-}}(r, z)$ and $p_{F_{+}}(r, z)$ are the distances of a point $P(r, \phi, z)$ on the surface of the spheroid from each of the two foci, $d_{F}$ is the distance between the foci and $\gamma$ is the length of an ellipse having the same major and minor axes of the considered spheroid. All these quantities can be calculated from the parameters $a$ and $b$. Hence, the $\zeta$ coordinate represents, together with the $\phi$ coordinate, the position on the spheroid surface.

Metachronal propulsion in Paramecia has been commonly modelled as antipletic metachrony, which involves effective stroke and wave crest velocity in opposite directions 17] (see Figure 1(b)). This also leads to the microorganisms swimming in 
the opposite direction with respect to the direction of the wave propagation. In order to implement this behaviour in the model, the deformation of the surface of the spheroid can be expressed as

$$
\begin{aligned}
& \delta_{S \perp}(\zeta, \phi, t)=\alpha d_{\perp} \sin (k \zeta-\omega t-\phi) \\
& \delta_{S \|}(\zeta, \phi, t)=\alpha d_{\|} \cos (k \zeta-\omega t-\phi-\pi)
\end{aligned}
$$

where $d_{\perp}$ and $d_{\|}$are the amplitudes of perpendicular and tangential deformation, respectively, $k=2 \pi / \lambda$ and $\omega=2 \pi f$, being $\lambda$ and $f$ the wavelength and the frequency of the wave of deformation. The term $-\pi$ in (5) accounts for the difference in the direction between the stroke of cilia and the wave propagation. The term $-\phi$ accounts, instead, for a direction of propagation of the wave that is not parallel to the main axis of the spheroid (and to the stroke of cilia), reflecting an aspect of the specific mode of metachrony observed in the reference biological organism (i.e. dexiopletic metachrony) [17]. The coefficient $\alpha$ accounts for the loss of coordination between cilia in the proximity of the extremities of the body of the actual microorganisms. It is represented by the arbitrary function $1-(|z| / a)^{3}$, which assumes a value of 0 at the extremities of the spheroid $(z= \pm a)$ and progressively raises to 1 when approaching the equatorial circumference of the spheroid $(z=0)$, leading to a deformation amplitude that rapidly becomes null towards the extremities of the spheroid.

The deforming surface of the micro-swimmer was treated as a moving wall. This was obtained by setting at the boundary the no-slip condition and the following components of the velocity of movement of the surface:

$$
\begin{aligned}
& \nu_{S \perp}(\zeta, \phi, t)=-\alpha d_{\perp} \omega \cos (k \zeta-\omega t-\phi) \\
& \nu_{S \|}(\zeta, \phi, t)=\alpha d_{\|} \omega \sin (k \zeta-\omega t-\phi-\pi)
\end{aligned}
$$

Equations (6) and (7) describe the perpendicular and tangential components, respectively, of the deformation velocity with respect to the non-deformed surface of the spheroid, and are obtained as the time-derivatives of (4) and $\sqrt{5}\left(\nu_{S \perp}=\dot{\delta}_{S \perp}\right.$ and $\left.\nu_{S \|}=\dot{\delta}_{S \|}\right)$.

The deformation of the surface described by the conditions in (6) and (7) is responsible for the establishment of the velocity and pressure fields $\boldsymbol{u}$ and $p$ in the fluid. The fluid, in turn, exerts a hydrodynamic force and torque on the body of the micro-swimmer, which can be found by integrating over its deformed surface $S^{\prime}$, as follows:

$$
\begin{aligned}
& \boldsymbol{F}_{H}=\int_{S^{\prime}} \boldsymbol{\sigma} \cdot \hat{\boldsymbol{n}} \mathrm{d} S^{\prime} \\
& \boldsymbol{T}_{H}=\int_{S^{\prime}} \boldsymbol{P}^{\prime} \times(\boldsymbol{\sigma} \cdot \hat{\boldsymbol{n}}) \mathrm{d} S^{\prime}
\end{aligned}
$$

where $\hat{\boldsymbol{n}}$ is the unit normal to $S^{\prime}$ into the fluid and $\boldsymbol{P}^{\prime}$ denotes positions on $S^{\prime}$. The action of the fluid is contained in the variable $\boldsymbol{\sigma}$, which represents the stress tensor given by

$$
\boldsymbol{\sigma}=-p \mathbf{I}+\eta_{f}\left[\nabla \boldsymbol{u}+(\nabla \boldsymbol{u})^{T}\right]
$$




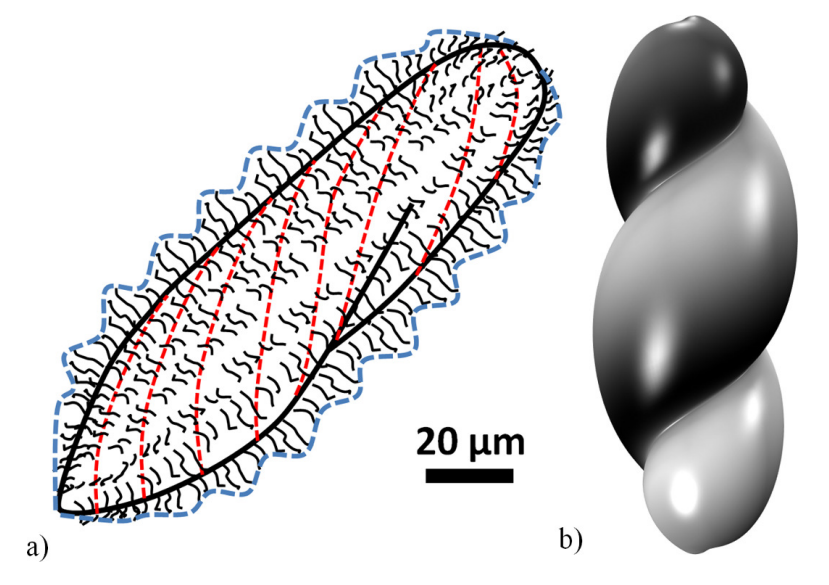

Figure 2. Modelling of the propulsion of Paramecium caudatum: a) schematic drawing of the microorganism emphasizing the distribution of cilia and the appearance of metachronal waves; b) the microorganism is modelled as a prolate spheroid with a continuous deforming surface mimicking the phenomenon of metachronal waves (the grayscale colour range represents the tangential component of deformation $\delta_{S \|}$

where $\mathbf{I}$ is the identity matrix [1]. The hydrodynamic actions of $(8)$ and $(9)$ account for both the propulsive and the viscous resistive actions exerted by the fluid on the body of the micro-swimmer. Because of the inherent symmetries of the problem, only the $z$-components of the two hydrodynamic actions are not null, resulting in a linear and an angular accelerations, $\ddot{z}$ and $\ddot{\phi}$, of the micro-swimmer. These can be obtained by

$$
\begin{aligned}
& F_{H, z}=m \ddot{z} \\
& T_{H, z}=J_{z} \ddot{\phi}
\end{aligned}
$$

where $m$ is the mass of the micro-swimmer and $J_{z}$ is its moment of inertia with respect to the $z$-axis. The linear and angular velocities of the micro-swimmer can thus be obtained respectively as $v_{m s}=\dot{z}$ and $\omega_{m s}=\dot{\phi}$.

It should be noticed that the model was defined using a reference system fixed on the micro-swimmer. Consequently, with respect to this moving frame, the microrobot is steady while the fluid goes toward it with an apparent velocity described by the linear and angular components $v_{f}=-v_{m s}$ and $\omega_{f}=-\omega_{m s}$. These velocities were thus considered for describing the velocity field condition set at the inlet boundary. Moreover, in order to model the apparent motion of the fluid with respect to the micro-swimmer, the external force term $\boldsymbol{f}_{e}$ of $(1)$ is derived from the accelerations in (11) and (12). This way, even if defined with respect to the frame fixed on the micro-swimmer, the model can accurately predict the effect of the resistive drag due to the movement of the microswimmer itself through the stress tensor $\boldsymbol{\sigma}$. As a result, the force and torque (and the related linear and angular accelerations) of (11) and (12) become almost zero after a short transient. 
Table 1. Values of the main input parameters adopted in the model. For some of them the actual biological value is adopted, while, for the others, values falling in the typical ranges of variation were used.

\begin{tabular}{lll}
\hline & $\begin{array}{l}\text { Paramecium } \\
\text { caudatum }\end{array}$ & 17. 24 \\
& $\begin{array}{l}\text { Modelled } \\
\text { micro-swimmer }\end{array}$ \\
\hline$a(\mu \mathrm{m})$ & 83 & 83 \\
$b(\mu \mathrm{m})$ & 20 & 20 \\
$d_{\perp}(\mu \mathrm{m})$ & $<6$ & 4.8 \\
$d_{\|}(\mu \mathrm{m})$ & $>d_{\perp}$ & 9.6 \\
$f(\mathrm{~Hz})$ & 40 & 40 \\
$\lambda(\mu \mathrm{m})$ & $10^{1}-10^{2}$ & 80 \\
\hline
\end{tabular}

\subsection{Preliminary validation}

The developed model was validated with biological data by predicting the swimming speed of a particular microorganism, Paramecium caudatum, that is schematically represented in Figure 2(a). This microorganism was selected because it is widely studied in biological experimental works. Nevertheless, not all the parameters needed for this modelling work are usually measured, and, if so, they are often extracted in different and particular conditions. For Paramecium caudatum we found in [24] a set of values including dimensions $a$ and $b$, beating frequency of cilia $f$ (corresponding to the frequency of the travelling-wave deformation) and speed of motion $v_{P c}$. The values of other parameters, such as amplitudes of deformation $d_{\perp}$ and $d_{\|}$and the wavelength $\lambda$, were chosen within their typical ranges of variability generally found in Paramecia. In particular, the peak-to-peak amplitude of perpendicular deformation should be smaller than the length of cilia, such that $2 d_{\perp} \leq 12 \mu \mathrm{m}$. In addition, considering the selected value of $d_{\perp}$, an estimation of the value of $d_{\|}$can be obtained by basic geometric considerations. For what concerns the wavelength $\lambda$, its values in Paramecia are reported to be in the tens-of-micrometres range [17], depending also on the viscosity of the specific fluid. The set of the parameters values adopted in this simulation is reported in Table 1.

The model described in Section 3.1 was implemented in a three-dimensional (3D) FE formulation by means of the commercial software COMSOL Multiphysics. A transient, time-dependent analysis was performed with a duration of 1.1 periods $T=f^{-1}$ of the waving deformation. Additional information about the implementation of the FE analysis can be found in the Supplementary Material, available on-line. The results show that, in the early part of the simulation (about $0.1 T$ ), the swimming speed readily increases, quickly achieving a quasi-steady value. The final speed of the modelled microswimmer was thus calculated as the average over a full period of propulsion (time $t$ ranging from $0.1 T$ to $1.1 T$ - see Supplementary Video 1, available on-line).

In Figure 3 a frame from the simulation is reported. The micro-swimmer, which mimics Paramecium caudatum according to Table 1, is represented by the deformed spheroid in the middle of the plot, with the tangential component of the deformation 


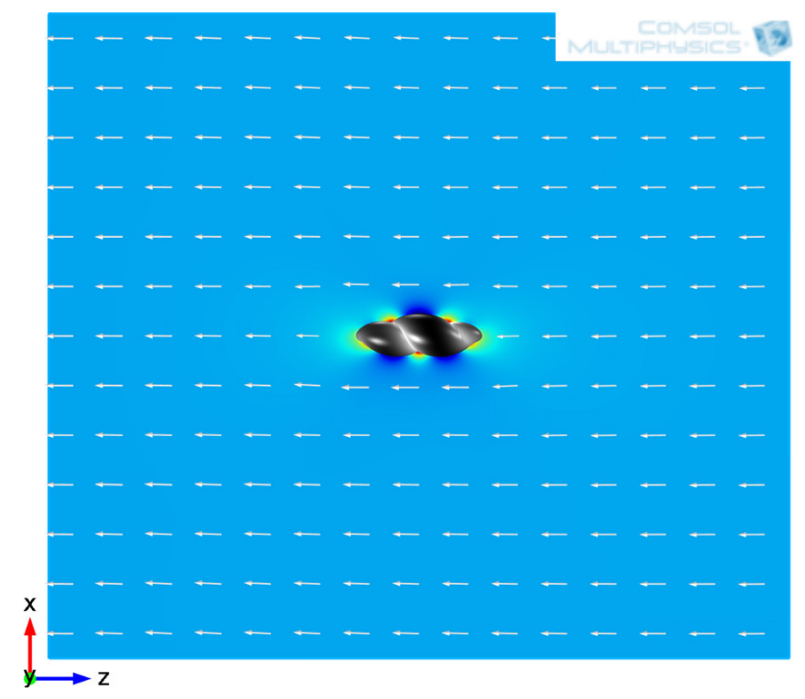

Figure 3. A frame from the simulation of the swimming of Paramecium caudatum, modelled as a prolate spheroid with a travelling-wave deforming surface (gray-scale, in the centre); the in-plane components of the relative fluid velocity are represented in $x z$ plane as both the colour-scale and white arrows. The final speed of the micro-swimmer is $-1.132 \mathrm{~mm} / \mathrm{s}$, corresponding to about 6.8 bodylengths $/ \mathrm{s}$.

$\delta_{S \|}$ represented in gray-scale on its surface as in Figure $2(\mathrm{~b})$. The colour-scale on the $y z$-plane is relative to the fluid velocity ( $z$-component), also represented by the inplane white arrows ( $x$ - and $z$-components). The final swimming speed of Paramecium caudatum predicted by the model was $-1.132 \mathrm{~mm} / \mathrm{s}$ ( 6.8 bodylengths $/ \mathrm{s})$. While the qualitative behaviour of the micro-swimmer is well predicted by the model (e.g. the negative sign of the velocity is a consequence of the anipletic metachrony), from a quantitative point of view the predicted velocity is slightly lower than the value found in the biological literature $(1.45 \mathrm{~mm} / \mathrm{s})[24]$. This is due to the fact that the assumptions underlying the envelope model are more likely to hold for the case of sympletic metachrony than for antipletic metachrony, since in the latter case cilia are more isolated during the "power" stroke [17].

\section{Role of the key features}

The analysis reported in this Section was performed for understanding the role of the key features of the propulsion mechanism based on metachronal waves. In order to actually achieve an artificial implementation of such a mechanism in a self-propelled microrobot, the mechanism itself must be simplified as much as possible with respect to the biological model. However, the effects of these simplifications on the microswimmer speed must be estimated, so that the features that are most responsible for the propulsion performance are maintained in the artificial implementation while the non-essential features are removed. The investigations provided in the following were conducted through FE modelling with COMSOL Multiphysics (additional information 


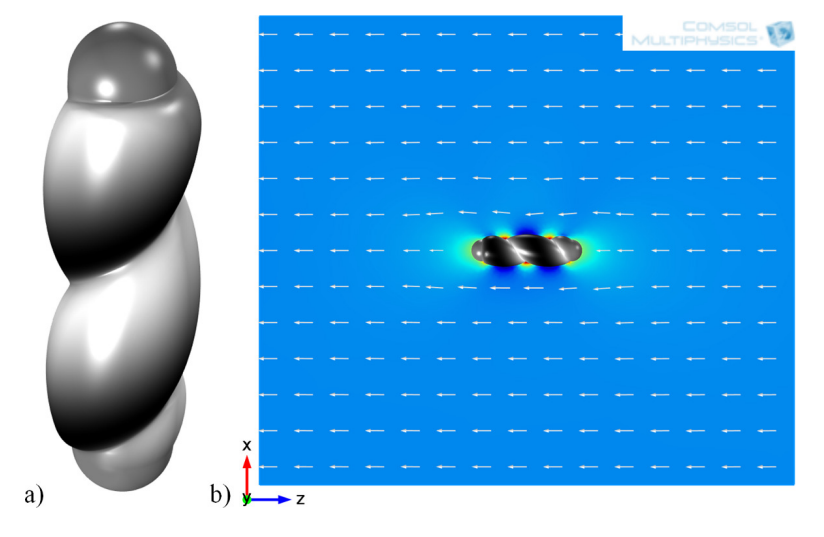

Figure 4. Simulation of the propulsion of an artificial micro-swimmer with a simplified cylindrical geometry: (a) shape with surface deformation; (b) a frame from the simulation. The predicted swimming speed is $-1.055 \mathrm{~mm} / \mathrm{s}$, corresponding to 5.3 bodylengths/s.

can be found in the Supplementary Material, available on-line.

\subsection{Shape}

First, the effect on the performance of simplifying the shape from a prolate spheroid to a cylinder with two hemispheres at the front and rear was investigated (see Figure 4(a)). Such a simplification would facilitate the fabrication of the artificial microrobots. In this analysis, only the lateral surface of the main cylindrical part of the micro-swimmer was considered as a deforming surface. This active part of the hypothetical microswimmer was assumed to have about the same length, $l_{c y l}$, of the total length of the previously analysed biological model and the same radius, $r_{c y l}$, of the maximum radius of the spheroid, such that $l_{c y l}=160 \mu \mathrm{m}$ and $r_{c y l}=20 \mu \mathrm{m}$. Moreover, the front and rear hemispheres have radius $r_{h e m i}=r_{c y l}$, resulting in a total length $l_{\text {tot }}=200 \mu \mathrm{m}$ for the envisioned artificial micro-swimmer. All the values of the parameters relating to the travelling wave deformation $\left(d_{\perp}, d_{\|}, \lambda\right.$ and $\left.f\right)$ were kept as in Table 1 , and we assumed $\alpha=1$ (i.e. a constant amplitude of deformation) along almost the entire active surface and rapidly becoming 0 close to the hemispherical ends.

In Figure 4(b) a frame is reported from the simulation of the micro-swimmer with the simplified cylindrical shape (see Supplementary Video 2, available on-line). The obtained swimming velocity is $-1.055 \mathrm{~mm} / \mathrm{s}$, corresponding to 5.3 bodylengths/s. With respect to the reference biological model, the change of shape led to a difference in performance of $-6.8 \%$. Therefore, we can reasonably assert that the particular geometry is not an essential feature in the propulsion of the micro-swimmer, hence the simplification can be adopted without a substantial reduction in performance. 


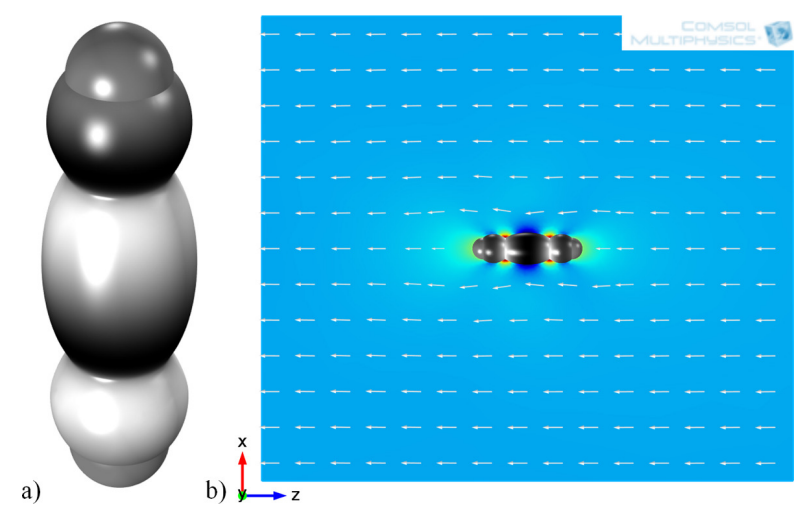

Figure 5. Simulation of the propulsion of an artificial micro-swimmer without the rotational behaviour: (a) shape with surface deformation; (b) a frame from the simulation. The predicted swimming speed is $-1.199 \mathrm{~mm} / \mathrm{s}$, corresponding to 6.0 bodylengths/s.

\subsection{Rotation}

Considering the cylindrical shape, as selected in Section 4.1, the second aspect investigated in this analysis is the rotational behaviour of the micro-swimmer. This is obtained as an effect of the phase shift introduced by the term $-\phi$ in equations (4) - (7), which leads to an actual direction of propagation of the travelling waves of deformation that is not parallel to the main axis of the micro-swimmer. This, indeed, is responsible for the arising of a non-zero hydrodynamic torque that makes the micro-swimmer rotate in the fluid. Therefore, the rotational behaviour can be removed by simply removing the phase shift term $-\phi$ from the equations describing the surface deformation and its velocity (see Figure 5(a)). Such a simplification would also lead to a simplified fabrication process.

The speed predicted by the model of the non-rotating micro-swimmer is $1.199 \mathrm{~mm} / \mathrm{s}$, which corresponds to 6.0 bodylengths/s. This configuration thus enables an enhancement of performance of $13.65 \%$ with respect to the rotating one. This result suggests this cylindrical non-rotating configuration as having a slightly better efficiency than the rotating one in translating the travelling-wave surface deformation in translational motion. A frame from the simulation of the non-rotating micro-swimmer (see Supplementary Video 3, available on-line) is reported in Figure 5(b).

\subsection{Deformation}

Considering the above results, we selected the cylindrical non-rotating configuration for the simulated implementation of a micro-swimmer. However, it is unlikely that the biological combination of tangential and perpendicular deformation could be replicated in an artificial device. In particular, the issue is related to the achievement of the specific combination of amplitudes and phase differences. Therefore, the final part of this analysis deals with the investigation of the performance of the micro-swimmer when 


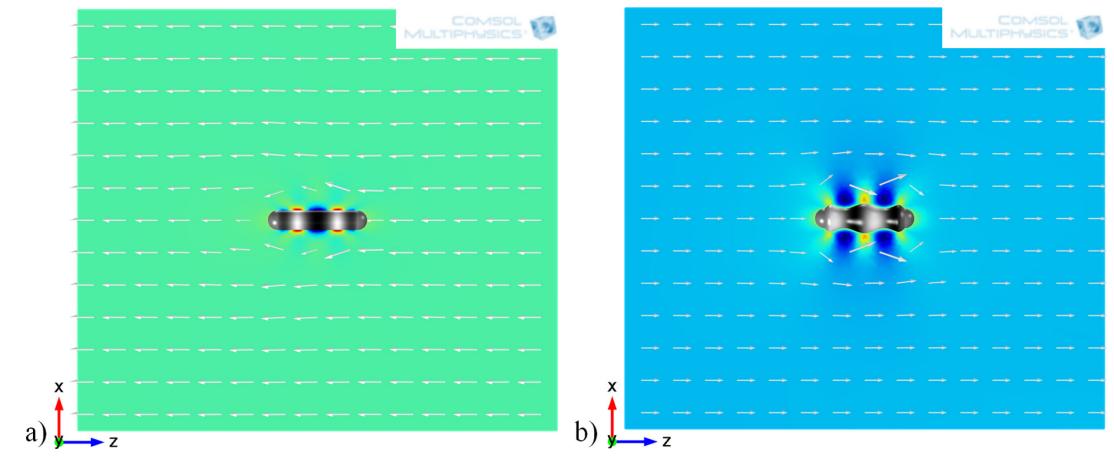

Figure 6. Effect of the two components of deformation velocity on the propulsion of the micro-swimmer: (a) tangential component, resulting in a swimming speed of $-0.133 \mathrm{~mm} / \mathrm{s}$, corresponding to 0.67 bodylengths/s; (b) perpendicular component, leading to a swimming speed of $0.091 \mathrm{~mm} / \mathrm{s}$, corresponding to 0.46 bodylengths $/ \mathrm{s}$.

employing only either the tangential or the perpendicular components of the deformation velocity. In order to provide a better comparison between the results, the amplitudes of deformation $d_{\perp}$ and $d_{\|}$were both set to $5 \mu \mathrm{m}$.

The results show that employing only the tangential component gives much better propulsion performance $(+46 \%)$ than the perpendicular component only. The resulting swimming speeds were $-0.133 \mathrm{~mm} / \mathrm{s}$ ( 0.67 bodylengths $/ \mathrm{s})$ for the tangential component (see Figure 6(a) and Supplementary Video 4) and $0.091 \mathrm{~mm} / \mathrm{s}$ (0.46 bodylengths/s) for the perpendicular component (see Figure 6(b) and Supplementary Video 5). It is noteworthy that both of them have much lower performances (about one order of magnitude) than that obtained through their biological combination.

One potential advantage of the perpendicular mode is that the amplitude and wavelength of the deformation can be tuned almost independently in order to maximize the swimming performance. This cannot be done in the tangential mode, where the wavelength strictly constrains the maximum possible value of the wave amplitude. In a previous work [16] we investigated the influence of the wave parameters (i.e. frequency, amplitude and wavelength) on the swimming velocity of a perpendicular travellingwave-based mechanism of propulsion. Those results qualitatively confirmed the trends obtained through the analytic envelope model [17], demonstrating that performances can be significantly enhanced by increasing the frequency and/or the wave amplitude and by decreasing the wavelength. Depending on the selected actuation technology and on the fabrication process, optimal configuration of these parameters can be selected in order to maximize the swimming speed of the artificial microrobot.

\section{Design and expected performance}

The analysis of the biological reference behaviour and the investigation on the contribution of its main features, respectively reported in Section 3 and Section 4 , provides the basic requirements for the conceptual design of a bioinspired propulsion 


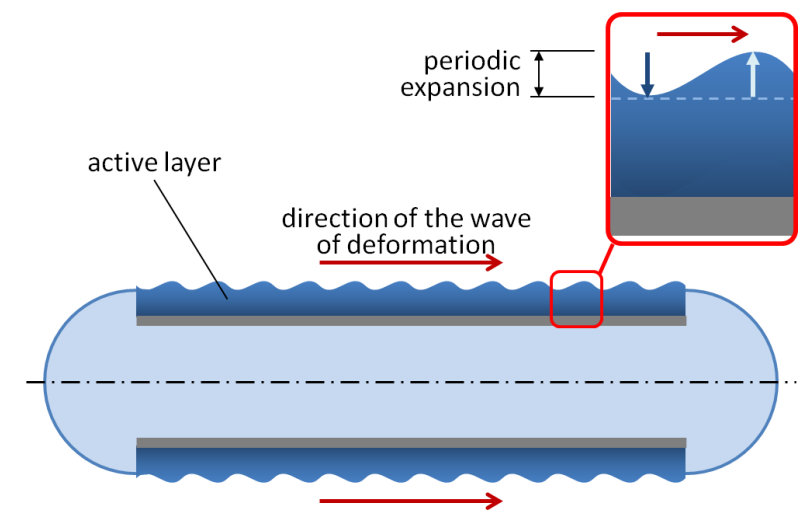

Figure 7. Concept of the propulsion system embedded in a swimming microrobot: the system is based on the travelling wave-shaped perpendicular deformation of the surface that is actuated by a controlled layer of active material.

system for a self-propelled swimming microrobot (Figure 7) and of the microrobot itself. We use the wording "bioinspired propulsion system" to refer to a microrobot component or subsystem that provides the microrobot with the bioinspired propulsion mechanism defined in Section 4 by means of embedded actuation. The requirements obtained through the previous analysis are:

- microrobot with cylindrical shape (see Section 4.1);

- propulsion system based on waves of deformation travelling longitudinally along the main axis of the microrobot (non-rotating behaviour - see Section 4.2);

- propulsion system exploiting the periodic, local perpendicular expansion/contraction of the surface (see Section 4.3).

In addition, we envision a microrobot of size ranging between $200 \mu \mathrm{m}$ (the size adopted in the simulations in Section 4) and $2 \mathrm{~mm}$ in length, with the radius varying accordingly. In a previous work, we proved that the linear speed of the micro-swimmer increases linearly with its size, keeping a constant relative velocity (bodylengths/s) [16]. Moreover, the wave parameters $\left(f, d_{\perp}\right.$ and $\left.\lambda\right)$ can be varied, as well, with respect to those extracted from the biological reference example and adopted in the simulations, affecting the micro-swimmer performance according to the trends in 16 .

Hence, the propulsion system will consist of a layer of active material (actuation element) enveloping the microrobot body and exhibiting a controlled wave-shaped perpendicular deformation that travels from the front to the rear side of the microrobot. Therefore, the actuation element represent the technological core of the proposed propulsion system, determining its actual feasibility and its performances.

The technological implementation of the actuation element is made plausible by the peculiar features of the proposed bioinspired propulsion mechanism. In particular, many state-of-the-art active materials can be produced as layers at the scale envisioned and guarantee controllable perpendicular expansion and contraction. In light of this, a micrometre-sized controllable rotary motor, which would be required by bacteria- 
inspired propulsion, or the continuous actuation system distributed in long and thin filament, as instead needed in eukaryotic-flagella-inspired propulsion, are, in our opinion, more unlikely to be achieved than the propulsion system proposed in this work.

In this context, the selection of the active material constituting the actuation and core element of the bioinspired propulsion system represent a crucial design step, which is addressed in the following.

\subsection{Selection of active material}

As we pointed out, the selection of the constitutive material for the active layer is a key aspect in the design of the bioinspired propulsion system and, as a consequence, of the microrobot as a whole.

The ideal active material for such an embodiment should have the following basic characteristics:

- controllable perpendicular expansion, possibly large and fast;

- electrically driven using low voltage;

- low power consumption;

- possibility to be operated in aqueous electrolytes;

- low weight.

The crucial requirement for the active material consists therefore in a perpendicular expansion that could be controlled by electrical signals. This would enable an easier control and a simplified fabrication.

Since the propulsion system is proposed for being implemented in a mobile and untethered microrobot, the requirements related to power and voltage are also particularly important. Not only the power consumption should be low enough to enable long operation with an on-board supply, but also the required voltage should be kept to a level that could be supplied by a simple component embedded within the microrobot body, such as a miniaturized battery or a tiny fuel cell.

The condition on the operation in aqueous electrolytes derives from the consideration that the envisioned microrobot, endorsed with the proposed propulsion system, will be used in medical applications and thus will swim in bodily fluids.

Low weight is instead needed for achieving quasi-neutral buoyancy of the whole microrobot. This feature simplifies the propulsion and the control of the microrobots [25], leading also to reduce power consumption.

5.1.1. Short overview of suitable actuation technologies. Electroactive polymers (EAPs) are often proposed for soft robotic applications, being intrinsically light-weight, compliant, silent and probably having lower fabrication costs than traditional actuators. Moreover, these soft actuators are inherently well suited for miniaturization, since they do not involve complex mechanisms and gearboxes. 
Propulsion of swimming microrobots inspired by metachronal waves

Table 2. Comparison among state-of-the-art electroactive polymers (EAPs) for their adoption as core active materials in the proposed propulsion system. For more details regarding the different EAP technologies we refer to [26 29]

\begin{tabular}{lllll}
\hline & DEs & Gels & IPMCs & CPs \\
\hline perpendicular expansion & $\begin{array}{l}\text { medium-large } \\
(<30 \%[30)\end{array}$ & large & none, & medium-large \\
& fast & slow & only bending & $(<50 \%, 31,32$ \\
speed & slow-medium & slow-medium \\
driving voltage 26 & $\left(10^{3}-10^{5} \mathrm{~V}\right)$ & $(10 \mathrm{~V})$ & $(1 \mathrm{~V})$ & $(1 \mathrm{~V})$ \\
& $60-90 \%$ & $30 \%$ & - & $<5 \%$ \\
efficiency 26 & allowed & inherent & allowed & inherent \\
operation in electrolytes & & & &
\end{tabular}

EAPs are generally classified in two groups: electronic EAPs, which are driven by electrostatic forces, and ionic EAPs, which are driven by an electrically induced transport of ions and/or solvent [33] (see Table 2). Electronic EAPs, such as dielectric elastomers deliver large strains, but are generally driven by high potentials $(\backsim \mathrm{kVs})$ making them substantially unsuitable for being adopted in untethered microrobots 34 . Ionic EAPs are generally wet and require low driving potentials. They comprise the subgroups gels, Ionic Polymer-Metal Composites (IPMCs), and conducting polymers (CPs). Gels are driven by a solvent swelling that is usually induced by an external $\mathrm{pH}$ change, but electronically triggered gels have also been described 35]. Moreover, they can be microfabricated [36, 37]. However, because they are driven by a solvent swelling effect, they are generally slow, meaning that they would fail to give any significant propulsion. IPMCs, instead, can only deliver a bending motion [38, 39], so they are unsuited for this application, as well.

An interesting candidate for generating travelling-wave deformation, however, is the conducting polymer polypyrrole $(\mathrm{PPy})$. PPy has not only been used as the active material in large actuators [40], but also been micro-fabricated [41 45], amongst others, to drive a microrobotic arm [46]. Being a polymer, PPy is intrinsically light-weight (about $1.5 \mathrm{~g} / \mathrm{cm}^{3}[47]$ ), thus easing the task of achieving quasi-neutral buoyancy of the whole microrobot. Moreover, it has low power consumption and, importantly, low operating voltages (1-2 V), so that it could be driven by an onboard power-supply [48]. PPy can be operated in almost any electrolyte, and has been shown to be biocompatible [49]. The volume change of PPy is based on a reversible electrochemical oxidation/reduction that drives a flow of ions [40] into or out of the material, combined with osmotic swelling [50]. A specifically interesting variant of $\mathrm{PPy}$ for implementing the proposed propulsion mechanism is PPy doped with the large anion dodecylbenzensulfonate, $\mathrm{PPy}(\mathrm{DBS})$, which has a large perpendicular volume change $31,32,51$.

5.1.2. Current performances of $P P y(D B S)$. When $P P y(D B S)$ is reduced to the neutral state, $\mathrm{Na}^{+}$ions, including their hydration shell, enter the polymer matrix and the 
material expands. When $\mathrm{PPy}(\mathrm{DBS})$ thereafter is oxidized, the $\mathrm{Na}^{+}$ions leave the matrix and the material shrinks. The perpendicular expansion profile comprises two parts. The first part of the expansion is fast, fairly linear and corresponds to the insertion of ions (including their hydration shell), while the second, slower part corresponds to the swelling by osmotic solvent intake [31,52]. Perpendicular strains up to $50 \%$ for $1 \mu \mathrm{m}$ films or an absolute expansion of $3.5 \mu \mathrm{m}$ for $10 \mu \mathrm{m}$ thick films have been reported (Carlsson et al. report absolute expansions up to $\sim 20 \mu \mathrm{m}$, but it is unclear what the initial PPy thickness was, probably 40-55 $\mu \mathrm{m}$ [31, 51]).

The full expansion of $\mathrm{PPy}(\mathrm{DBS})$, however, can only be reached when operating it at very low frequencies, thus allowing the slow osmotic swelling to take place. For the range of frequencies required for the actuation of the bioinspired microrobot, only the first, linear part of the expansion profile will be exploited, corresponding to the fast ionintake. To the best of our knowledge the fastest large expansions have been presented by Carlsson et al. [51]. By optimizing the expansion with respect to the electrolyte concentration, ionic species, temperature and applied actuation potential, they were able to achieve a speed of $7.4 \mu \mathrm{m} / \mathrm{s}$ for the linear part of the expansion profile.

\subsection{Expected performance of the conceptual artificial micro-swimmer}

Considering the maximum expansion and contraction speed found in literature, under the assumption of exploiting the fast linear part of the expansion/contraction profile, we set the average perpendicular deformation rate for PPy at $\bar{\nu}_{S \perp}^{P P y} \simeq 8 \mu \mathrm{m} / \mathrm{s}$. This parameter gives a representation of the effect of both the amplitude $d_{\perp}$ and the frequency $f$ of the wave of deformation on the final performances of the microswimmer, as preliminarily addressed in [16]. Even if performance improvements could be obtained by optimizing the trade-off between amplitude and frequency of deformation of PPy, this average deformation rate allowed us to obtain an estimate of the order of magnitude of the propulsion speed, which was about $1 \mu \mathrm{m} / \mathrm{s}$ (corresponding to about 0.05 bodylengths/s).

The huge difference in performance between the modelled ideal condition based on the Paramecium (resulting in a swimming velocity of the order of hundreds of micrometers per seconds) and the envisioned design based on $\operatorname{PPy}(\mathrm{DBS})(\backsim 1 \mu \mathrm{m} / \mathrm{s})$ can be understood when we consider the performance of the ideal actuator implied in the model. The average perpendicular deformation rate adopted in the simulations reported in Section 4, as extrapolated from the biological reference model, is $\bar{\nu}_{S \perp}^{b i o}=$ $4 d_{\perp} f=600 \mu \mathrm{m} / \mathrm{s}$ (see Table 1 ). This value is two orders of magnitude larger than that achievable with current PPy-based actuators.

\section{Conclusions}

In this paper, a propulsion mechanism inspired by that occurring in ciliates was proposed for self-propelled mobile microrobots. Ciliates were chosen as a reference biological 
model because they are the largest and fastest biological swimmers at the microscale.

Considering that their propulsion performances are mostly due to a self-emerging collective behaviour of cilia, known as metachronal waves, the proposed artificial mechanism of propulsion aimed at mimicking this high-level behaviour, rather than the individuality of the thousands of cilia covering the surface of a single microorganism.

With this choice we pursued a design of a propulsion system much simpler than the real biological mechanism of interest. Given that actually embedding a propulsion system in a mobile self-propelled microrobot puts additional constraints on its design, compared to microrobots propelled by external means such as magnetic fields, a high level of simplification is essential to enable such an implementation.

The biological mechanism of propulsion was investigated by means of a finiteelements model that considered surface deformations of the micro-swimmer that have features resembling metachronal waves in Paramecium caudatum, predicting a swimming speed close to the biological one. This preliminary validation also proves that the metachronal wave principle/mechanism is indeed the major feature of ciliary locomotion, giving additional support to our choice of taking inspiration from this key high-level behaviour, rather than individually replicating cilia and their complex beating pattern.

Aiming at maximizing the chances of the propulsion mechanism to be implemented in a swimming microrobot, we also investigated generalizations that could be adopted in the bioinspired design process in order to relax the technological requirements for the propulsion system. These relates to the shape of the micro-swimmer, its rotating behaviour and to the role of the components (tangential and perpendicular) of the surface deformation. This analysis allowed us to verify that even the simplest of the investigated configurations (cylindrical body, non-rotating behaviour, perpendicular deformation) is propulsive, and also to estimate the swimming performance that could be expected for an artificial micro-swimmer.

Subsequently, the actual feasibility of the bioinspired propulsion mechanism, obtained through these sequential simplifications, was addressed. In particular, we analyzed the state-of-the-art actuation technologies, selecting the most suitable one (the electroactive polymer polypyrrole - PPy) considering the constraints derived from the embodiment of the propulsion system in a self-propelled microrobot. However, the analysis unveiled the inadequacy of the current actuation performance of this technology in terms of expansion rates, which resulted in a very low predicted propulsion speeds of the envisioned artificial micro-swimmer.

In order to achieve a practical propulsion, a speed of 0.5 bodylengths/s (i.e. $0.1-$ $1 \mathrm{~mm} / \mathrm{s}$, depending on the microrobot size) is needed and the target specifications for the electroactive material to be met are: expansions in the order of $10 \mu \mathrm{m}$ at frequencies of $10 \mathrm{~Hz}$. More generally, this work emphasizes the major role that investigation on new microscale actuation technologies have in microrobotics. We provide suggestions for research in materials and designs of micro-actuators to pursue an effective embodiment in a mobile sub-millimetre bioinspired swimming robotic device. In particular, we believe that, since the performances of EAPs have been traditionally optimized for 
macroscale and/or tethered embodiments, the research on self-propelled microrobots could guide and drive new research on these smart materials by suggesting new directions of development.

\section{References}

[1] E. Lauga and T.R. Powers. The hydrodynamics of swimming microorganisms. Reports on Progress in Physics, 72(9):096601, 2009.

[2] J.J. Abbott, Z. Nagy, F. Beyeler, and B.J. Nelson. Robotics in the small, part I: Microbotics. Robotics $\&$ Automation Magazine, IEEE, 14(2):92-103, 2007.

[3] J.J. Abbott, K.E. Peyer, M.C. Lagomarsino, L. Zhang, L. Dong, I.K. Kaliakatsos, and B.J. Nelson. How should microrobots swim? The International Journal of Robotics Research, 28(11-12):1434-1447, 2009.

[4] B.J. Nelson, I.K. Kaliakatsos, and J.J. Abbott. Microrobots for minimally invasive medicine. Annual Review of Biomedical Engineering, 12(1):55-85, 2010.

[5] E.M. Purcell. Life at low reynolds number. American Journal of Physics, 45(1):3-11, 1977.

[6] S. Tottori, L. Zhang, F. Qiu, K.K. Krawczyk, A. Franco-Obregón, and B.J. Nelson. Magnetic helical micromachines: Fabrication, controlled swimming, and cargo transport. Advanced Materials, 24(6):811-816, 2012.

[7] L. Zhang, J.J. Abbott, L. Dong, B.E. Kratochvil, D. Bell, and B.J. Nelson. Artificial bacterial flagella: Fabrication and magnetic control. Applied Physics Letters, 94(6):064107-3, 2009.

[8] R. Dreyfus, J. Baudry, M.L. Roper, M. Fermigier, H.A. Stone, and J. Bibette. Microscopic artificial swimmers. Nature, 437(7060):862-865, 2005. 10.1038/nature04090.

[9] M. Roper, R. Dreyfus, J. Baudry, M. Fermigier, J. Bibette, and H.A. Stone. Do magnetic microswimmers move like eukaryotic cells? Proceedings of the Royal Society A: Mathematical, Physical and Engineering Science, 464(2092):877-904, 2008.

[10] A. Ghanbari, M. Bahrami, and M.R.H. Nobari. Methodology for artificial microswimming using magnetic actuation. Physical Review E, 83(4):046301, 2011. PRE.

[11] A. Babataheri, M. Roper, M. Fermigier, and O. Du Roure. Tethered fleximags as artificial cilia. Journal of Fluid Mechanics, 678:5-13, 2011.

[12] S.N. Khaderi, C.B. Craus, J. Hussong, N. Schorr, J. Belardi, J. Westerweel, O. Prucker, J. Ruhe, J.M.J. den Toonder, and P.R. Onck. Magnetically-actuated artificial cilia for microfluidic propulsion. Lab on a Chip, 11(12):2002-2010, 2011.

[13] B. Behkam and M. Sitti. Design methodology for biomimetic propulsion of miniature swimming robots. Journal of Dynamic Systems, Measurement, and Control, 128(1):36-43, 2006.

[14] K. Ehlers and J. Koiller. Micro-swimming without flagella: Propulsion by internal structures. Regular and Chaotic Dynamics, 16(6):623-652, 2011.

[15] E. Setter, I. Bucher, and S. Haber. Low-reynolds-number swimmer utilizing surface traveling waves: Analytical and experimental study. Physical Review E, 85(6):066304, 2012. PRE.

[16] S. Palagi, B. Mazzolai, and L. Beccai. Modeling of a propulsion mechanism for swimming microrobots inspired by ciliate metachronal waves. In Biomedical Robotics and Biomechatronics (BioRob), 2012 4th IEEE RAS \& EMBS International Conference on, pages 264-269, 2012.

[17] S. Childress. Mechanics of swimming and flying, volume 2. Cambridge University Press, 1981.

[18] B. Guirao and J.-F. Joanny. Spontaneous creation of macroscopic flow and metachronal waves in an array of cilia. Biophysical Journal, 92(6):1900-1917, 2007.

[19] S. Gueron and K. Levit-Gurevich. Energetic considerations of ciliary beating and the advantage of metachronal coordination. Proceedings of the National Academy of Sciences, 96(22):12240$12245,1999$.

[20] J. Elgeti and G. Gompper. Emergence of metachronal waves in cilia arrays. Proceedings of the National Academy of Sciences, 2013. 
[21] H. Machemer. Ciliary activity and the origin of metachrony in paramecium: Effects of increased viscosity. Journal of Experimental Biology, 57(1):239-259, 1972.

[22] J.R. Blake. A spherical envelope approach to ciliary propulsion. Journal of Fluid Mechanics, 46(01):199-208, 1971.

[23] M.J. Lighthill. On the squirming motion of nearly spherical deformable bodies through liquids at very small reynolds numbers. Communications on Pure and Applied Mathematics, 5(2):109-118, 1952.

[24] Y. Katsu-Kimura, F. Nakaya, S.A. Baba, and Y. Mogami. Substantial energy expenditure for locomotion in ciliates verified by means of simultaneous measurement of oxygen consumption rate and swimming speed. Journal of Experimental Biology, 212(12):1819-1824, 2009.

[25] S. Palagi, B. Mazzolai, C. Innocenti, C. Sangregorio, and L. Beccai. How does buoyancy of hydrogel microrobots affect their magnetic propulsion in liquids? Applied Physics Letters, 102(12):124102-5, 2013.

[26] P. Brochu and Q. Pei. Advances in dielectric elastomers for actuators and artificial muscles. Macromolecular Rapid Communications, 31(1):10-36, 2010.

[27] E.W.H. Jager and D. Carlsson. Electro-active polymer microactuators. Materials Science 8 Engineering R-Reports, 56:64-72, 2007.

[28] R. Pelrine, R. Kornbluh, J. Joseph, R. Heydt, Q. Pei, and S. Chiba. High-field deformation of elastomeric dielectrics for actuators. Materials Science and Engineering: C, 11(2):89-100, 2000.

[29] P. Sommer-Larsen and R. Kornbluh. Polymer actuators. In Actuator 2022, pages 371-378, Bremen, Germany, 2002.

[30] M. Jungmann and H.F. Schlaak. Electrostatic actuators with elastic dielectric for use on tactile displays. In Actuator 2002, pages 391-394, Bremen, Germany, 2002.

[31] D. Melling, S. Wilson, and E.W.H. Jager. The effect of film thickness on polypyrrole actuation assessed using novel non-contact strain measurements. Smart Materials $\mathscr{E}$ Structures, Accepted, 2013.

[32] E. Smela and N. Gadegaard. Surprising volume change in PPy(DBS): An atomic force microscopy study. Advanced Materials, 11(11):953-957, 1999.

[33] Y. Bar-Cohen. Electroactive polymer (EAP) actuators as artificial muscles: reality, potential, and challenges, volume 136. Society of Photo Optical, 2004.

[34] R. Pelrine, R. Kornbluh, Q. Pei, and J. Joseph. High-speed electrically actuated elastomers with strain greater than 100\%. Science, 287(5454):836-839, 2000.

[35] Y. Osada, H. Okuzaki, and H. Hori. A polymer gel with electrically driven motility. Nature, 355(6357):242-244, 1992. 10.1038/355242a0.

[36] Y. Ito. Photolithographic synthesis of intelligent microgels. Journal of Intelligent Material Systems and Structures, 10(7):541-547, 1999.

[37] D.J. Beebe, J.S. Moore, J.M. Bauer, Q. Yu, R.H. Liu, C. Devadoss, and B.-H. Jo. Functional hydrogel structures for autonomous flow control inside microfluidic channels. Nature, 404(6778):588-590, 2000. 10.1038/35007047.

[38] K. Onishi, S. Sewa, K. Asaka, N. Fujiwara, and K. Oguro. Biomimetic microactuators based on polymer electrolyte/gold composite driven by low voltage. In Micro Electro Mechanical Systems, 2000. MEMS 2000. The Thirteenth Annual International Conference on, pages 386-390, 2000.

[39] S. Nemat-Nasser and Y. Wu. Comparative experimental study of ionic polymer-metal composites with different backbone ionomers and in various cation forms. Journal of Applied Physics, 93(9):5255-5267, 2003.

[40] Q. Pei and O. Inganaes. Electrochemical applications of the bending beam method. 1. mass transport and volume changes in polypyrrole during redox. The Journal of Physical Chemistry, 96(25):10507-10514, 1992. doi: 10.1021/j100204a071.

[41] T.F. Otero, J. Rodriguez, E. Angulo, and C. Santamaria. Artificial muscles from bilayer structures. Synthetic Metals, 57(1):3713-3717, 1993.

[42] E. Smela, O. Inganäs, and I. Lundström. Controlled folding of micrometer-size structures. Science, 
268(5218):1735-1738, 1995.

[43] G. Alici, V. Devaud, P. Renaud, and G. Spinks. Conducting polymer microactuators operating in air. Journal of Micromechanics and Microengineering, 19(2):025017, 2009.

[44] E.W.H. Jager, E. Smela, and O. Ingans. Microfabricating conjugated polymer actuators. Science, 290(5496):1540-1545, 2000.

[45] A. Khaldi, C. Plesse, C. Soyer, E. Cattan, F. Vidal, C. Legrand, and D. Teyssie. Conducting interpenetrating polymer network sized to fabricate microactuators. Applied Physics Letters, 98(16):164101-3, 2011.

[46] E.W.H. Jager, O. Ingans, and I. Lundstrm. Microrobots for micrometer-size objects in aqueous media: Potential tools for single-cell manipulation. Science, 288(5475):2335-2338, 2000.

[47] Z. Qi and P.G. Pickup. Size control of polypyrrole particles. Chemistry of Materials, 9(12):29342939, 1997. doi: $10.1021 / \mathrm{cm} 970280 q$.

[48] T. Goettsche, A. Schumacher, J. Kohnle, S. Messner, and R. Zengerle. Highly integrated oral drug delivery system with valve based on Electro-Active-Polymer. In Micro Electro Mechanical Systems, 200\%. MEMS. IEEE 20th International Conference on, pages 461-464, 2007.

[49] E.W.H. Jager. Conjugated polymers as actuators for medical devices and microsystems. In J. Leger, M. Berggren, and S. Carter, editors, Iontronics: Ionic Carriers in Organic Electronic Materials and Devices. CRC Press, 2010.

[50] L. Bay, T. Jacobsen, S. Skaarup, and K. West. Mechanism of actuation in conducting polymers: Osmotic expansion. The Journal of Physical Chemistry B, 105(36):8492-8497, 2001. doi: 10.1021/jp003872w.

[51] D. Carlsson, E. Jager, M. Krogh, and M. Skoglund. Systems, device and object comprising electroactive polymer material, methods and uses relating to operation and provision thereof. Patent Application, 03 2009. WO 2009/038501 A1.

[52] M. Jafeen, M. Careem, and S. Skaarup. Speed and strain of polypyrrole actuators: dependence on cation hydration number. Ionics, 16(1):1-6, 2010. 